\section{Case Reports in Oncology}

\title{
Rapid Progressive Glioblastoma despite Radiation in a Patient with Myelodysplastic Syndrome
}

\author{
Eric T. Wong ${ }^{a}$ Justin Moore ${ }^{b}$ Lauren Hertan ${ }^{c}$ Erik J. Uhlmann ${ }^{a}$ \\ aBrain Tumor Center \& Neuro-Oncology Unit, Department of Neurology, Beth Israel \\ Deaconess Medical Center, Harvard Medical School, Boston, MA, USA; ${ }^{b}$ Division of \\ Neurosurgery, Department of Surgery, Beth Israel Deaconess Medical Center, Harvard \\ Medical School, Boston, MA, USA; 'Department of Radiation Oncology, Beth Israel \\ Deaconess Medical Center, Harvard Medical School, Boston, MA, USA
}

\author{
Keywords \\ Glioblastoma $\cdot$ Myelodysplastic syndrome $\cdot$ Immune dysfunction
}

\begin{abstract}
The rapidity of glioblastoma progression can be exacerbated by impaired systemic immune surveillance. We describe an elderly woman with advanced $5 q-$ myelodysplastic syndrome (MDS) associated with trilineage dysfunction in hematopoiesis. She also developed multiple solid tumor malignancies including ER/PR-positive and HER2-negative breast cancer, probable lung cancer without histologic confirmation, and primary glioblastoma with a high proliferation index of $80 \%$. Because of low platelet counts of $20,000-30,000 / \mu \mathrm{L}$ that required periodic transfusion and a reduced white cell count of $600-900 / \mu \mathrm{L}$, she was deemed unsafe to take concomitant daily temozolomide during radiation and her glioblastoma was treated with a shortened course of radiotherapy alone. Her baseline absolute neutrophil count was 110$390 / \mu \mathrm{L}$, and $\mathrm{CD} 4^{+}$and $\mathrm{CD} 8^{+}$lymphocyte counts were $235 / \mu \mathrm{L}$ and $113 / \mu \mathrm{L}$, respectively. During the last week of radiation, the patient developed a nonfluent aphasia, increased fatigue, and aspiration pneumonia. A gadolinium-enhanced head MRI, obtained 2 days after completion of radiation and 39 days after biopsy, demonstrated near tripling of the size of the left frontal tumor with a significant amount of adjacent cerebral edema. This case raises the possibility that advanced MDS is a negative immunomodulatory condition that can accelerate glioblastoma progression.




\section{Introduction}

Glioblastoma is invariably a fatal disease. Current treatment consists of maximum safe neurosurgical resection followed by radiation and concomitant temozolomide chemotherapy with or without tumor treating fields. This multimodality standard-of-care treatment results in a median overall survival of 14.6-20.9 months [1]. Bevacizumab is an accepted treatment for recurrent glioblastoma, entailing a median survival of 7.2-9.2 months [2]. However, these survival benchmarks can be influenced by concurrent systemic dysfunction within the patient. For example, it is now recognized that glioblastoma patients have both systemic and local immunosuppressive mechanisms that disable anti-tumor immunity [3]. Furthermore, temozolomide can cause severe pancytopenia in some patients, despite a documented low rate of neutropenia and thrombocytopenia of $7 \%$ and $12 \%$, respectively [4]. Therefore, poor bone marrow function could potentially limit the benefit of temozolomide when combined with radiation or in the adjuvant setting after radiotherapy in the multimodality treatment of glioblastoma patients.

Myelodysplastic syndrome (MDS) is a heterogeneous disorder of bone marrow failure with a propensity for transforming into acute myelogenous leukemia. Patient survival is variable, ranging from a median of 6 to 60 months, depending on the cytogenetic abnormalities [5]. We here report on an Asian woman with 5q- MDS, leukopenia, and initially nontransfusion-dependent thrombocytopenia who developed a left frontal glioblastoma. Although her marrow dysfunction precluded her from receiving standard-of-care daily temozolomide safely during radiotherapy, short-course radiation treatment alone had no effect in halting the growth of her tumor. This case indicates that a competent immune system in the host may be a prerequisite for radiation efficacy against glioblastoma.

\section{Case Description}

The patient was a 78-year-old right-handed Chinese woman who had developed slurred speech in early 2020; a noncontrast head CT showed hypodensity in the left frontal lobe of the brain. CT of the chest, abdomen, and pelvis showed growth of a mixed-density mass in the left upper lung, from $10 \times 22 \mathrm{~mm}$ to $15 \times 16 \mathrm{~mm}$ over 2 months, and a new peripherally located left lower lung nodule measuring $10 \times 16 \mathrm{~mm}$. On the following day, a gadoliniumenhanced MRI revealed that the intracranial mass was cystic with heterogeneously enhancing characteristics in the left frontal operculum, measuring $35 \times 37 \mathrm{~mm}$ and displaying the hallmarks of a primary brain tumor. A decision was made to obtain tissue confirmation from the cerebral mass in order to determine the histologic diagnosis of this malignancy, and a stereotaxic biopsy was performed. The pathology revealed glioblastoma, and immunohistochemical staining was negative for IDH-1 R132H mutation but positive for GFAP, Olig-2, and p53, suggesting a p53 mutation. The Ki-67 index showed $80 \%$ positivity, indicating a high proliferation rate. Biopsy of the lung nodules was deferred until her glioblastoma was treated and stabilized.

The patient also had a two-decade-long history of MDS. In 2004, she was found to have leukopenia and anemia. A bone marrow biopsy performed in 2009 showed hypercellular marrow with mild dyspoiesis. There were $1 \%$ blasts in the aspirate, and cytogenetic analysis of 18 out of 20 cells revealed an interstitial deletion of the long arm of chromosome 5q, indicating a diagnosis of MDS with $5 q$ deletion. The patient was asymptomatic at that time, and a decision was made to reserve treatment and observe her. In 2011, she experienced increased fatigue, shortness of breath, and episodic lightheadedness. Darbepoetin alfa was administered, but stopped after showing no improvement in hematocrit. The patient subsequently

\section{Karger'}



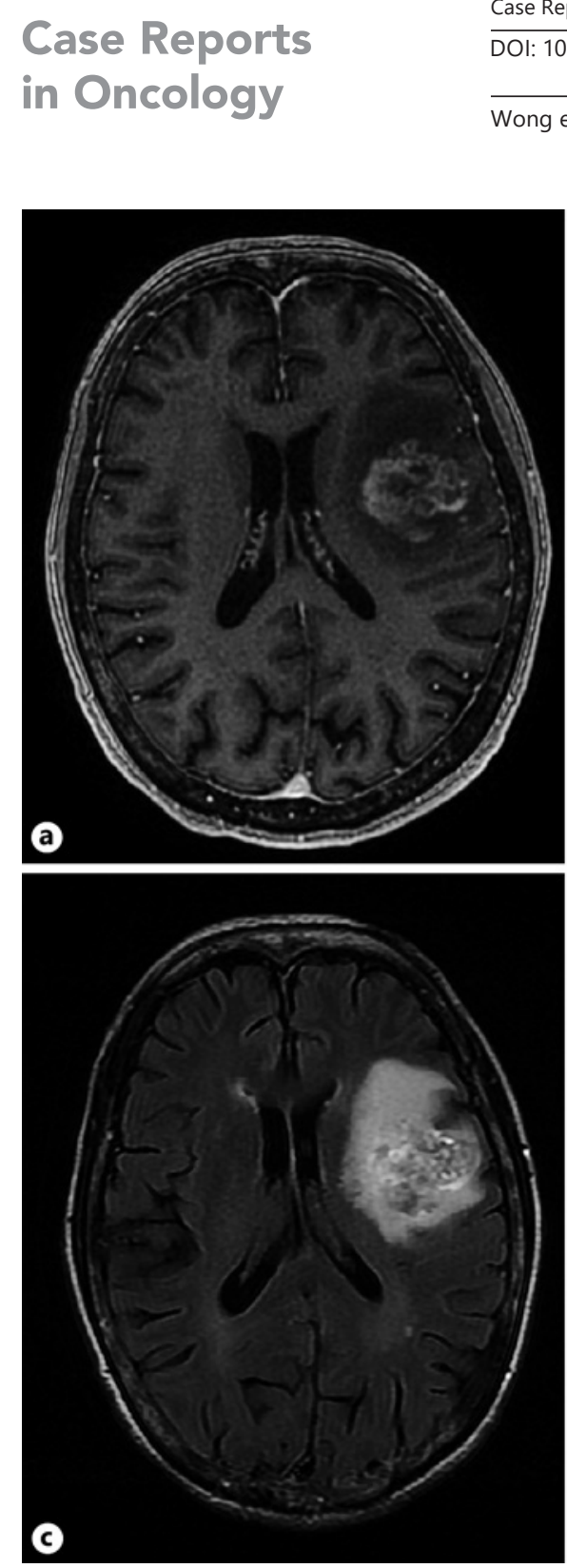

Fig. 1. Glioblastoma progression despite radiation treatment. The patient was diagnosed with an irregular, heterogeneously enhancing mass in the left frontal brain (a) associated with surrounding cerebral edema (c). A stereotaxic biopsy demonstrated glioblastoma with a high Ki-67 proliferation index of 80\%, and immunohistochemical staining was negative for IDH-1 R132H mutation but positive for GFAP, p53, and Olig-2. The post-radiotherapy head MRI showed a substantial increase in gadolinium enhancement (b) and cerebral edema (d).

received lenalidomide in 2012, but this was withheld after 2 months due to neutropenia. She was treated again with lenalidomide in 2017 , but it was discontinued shortly afterwards as a result of abdominal cramps and diarrhea. No additional treatment was attempted thereafter.

In addition, she had been diagnosed with stage II invasive breast carcinoma in the left breast while she had still been in China in 2005. She underwent a lumpectomy, and an invasive ductal carcinoma, measuring $0.6 \mathrm{~cm}$, was removed. Axillary lymph node dissection revealed that one of 13 axillary nodes was positive for carcinoma. The patient's tumor was ER and PR positive but HER 2 negative. She received radiation, followed by tamoxifen for 1 year and then letrozole for 4 additional years. 
After the diagnosis of glioblastoma, the patient started radiation 16 days after her brain biopsy and received 4,005 cGy over 15 fractions. No temozolomide was administered concurrently, due to leukopenia and thrombocytopenia with a platelet count of $20,000-30,000 / \mu \mathrm{L}$ and leukopenia with a total white cell count of 600-900/ $\mu \mathrm{L}$. Her absolute neutrophil count was $110-390 / \mu \mathrm{L}$, and $\mathrm{CD}^{+}$and $\mathrm{CD}^{+}$lymphocyte counts were $235 / \mu \mathrm{L}$ and $113 / \mu \mathrm{L}$, respectively. During the last week of radiation, the patient developed a nonfluent aphasia, wordfinding difficulty, increased fatigue, and opacity on chest X-ray thought to be caused by aspiration pneumonia. The patient was admitted to the hospital for management. A gadoliniumenhanced head MRI, obtained 2 days after completion of radiation and 39 days after biopsy, demonstrated near tripling of the size of the left frontal tumor to $52 \times 65 \mathrm{~mm}$ (Fig. 1). The patient was subsequently sent home with hospice care support and died 50 days after diagnosis of the glioblastoma.

\section{Discussion}

Successful treatment of a tumor using radiation requires healthy immune function in the patient. This type of anti-tumor immunity is initiated by the release of molecules known as damage-associated molecular patterns during tumor cell death and activation of an intratumoral type I interferon response [6, 7]. Similarly, the efficacy of certain chemotherapy drugs also depends on the contribution of type I interferon from immunogenic tumors [8]. Therefore, host immunity is an important prerequisite for effective anti-tumor response, and this effect may be increased further when radiation and chemotherapy are combined [8,9]. Indeed, for the treatment of newly diagnosed glioblastoma patients, the addition of temozolomide to radiotherapy improved their median overall survival. More importantly, the proportion of longer-term survivors improved from 11 to $27 \%$ at 2 years, 4 to $16 \%$ at 3 years, 3 to $12 \%$ at 4 years, and 2 to $10 \%$ at 5 years [10]. What was not captured in the trial data is the level of immune dysfunction in the glioblastoma cohorts, and this may ultimately impact their overall survival.

Glioblastoma patients have intrinsic immune suppression. Lymphopenia was present in nearly $25 \%$ of treatment-naïve and dexamethasone-naïve, newly diagnosed patients, compared to $11 \%$ of control patients without cancer [11]. Both $\mathrm{CD} 4^{+}$and $\mathrm{CD} 8^{+}$lymphocyte counts are low and, according to one study, $15 \%$ of those have $\mathrm{CD} 4^{+}$count $<200$ cells $/ \mu \mathrm{L}$, the threshold for defining AIDS in HIV-infected individuals $[11,12]$. Therefore, it is not a surprise that patients with a CD $4^{+}$count $<200$ cells $/ \mu \mathrm{L}$ die sooner than those with a $\mathrm{CD} 4^{+}$count $\geq 200$ cells/ $\mu \mathrm{L}$ despite treatment with radiotherapy and temozolomide [13]. They do not necessarily die of opportunistic infection per se, but their dysfunctional anti-tumor immunity is probably a major contributor to their earlier death. This earlier death rate can be further exacerbated by the use of systemic immunosuppressants such as dexamethasone or inhibitors of the mammalian target of rapamycin $[14,15]$.

There were a number of factors that predisposed our patient to a poor treatment outcome. First, she had advanced MDS with trilineage dysfunction in hematopoiesis. Temozolomide was contraindicated due to the patient's low white blood cell and platelet counts, therefore limiting her use of temozolomide during radiation and potentially attenuating her response to radiation treatment. Second, her MDS also severely affected her adaptive immune system, and this defect is characterized by low $\mathrm{CD}^{+}$and $\mathrm{CD} 8^{+}$lymphocyte counts. Although these counts were not below the threshold for severe immunodeficiency as in AIDS, they were still markers of her weakened adaptive immune function as a result of MDS and older age, both of which can attenuate the number of naïve T cells capable of antigen stimulation and expansion to effect an anti-glioblastoma immunity. Furthermore, these systemic conditions also predis-

\section{Karger'}


posed her to develop multiple primary malignancies secondary to impaired immune surveillance. Third, this patient was not a candidate for extensive brain surgery, due to her low platelet count in the range of $20,000-30,000 / \mu \mathrm{L}$. Because her thrombocytopenia was stemming from primary marrow failure, romiplostim and daily transfusions were unlikely to boost her platelets sufficiently to an acceptable level of safety for extensive tumor resection and the immediate postoperative period. Furthermore, a large glioblastoma contains a hypoxic microenvironment that is conducive to local immunosuppression. Although bevacizumab can alleviate increased vascular permeability, making the tumor and the surrounding brain less edematous and radiation potentially more effective, this medication can still cause hemorrhage within the tumor despite daily platelet transfusion. Lastly, lenalidomide is an effective medication for stabilizing MDS with $5 q$ deletion [16] and has antiangiogenic effects [17], making it ideal for stabilizing MDS and attenuating cerebral edema from the glioblastoma. Unfortunately, our patient developed intolerable abdominal cramps and diarrhea during her use of the medication. Collectively, all of these factors contributed to a poor outcome in this patient.

\section{Conclusions}

We described a patient with 5q- MDS who developed ER- and PR-positive but HER2negative breast cancer, probable lung cancer on imaging, and biopsy-proven glioblastoma. She progressed clinically despite cranial irradiation, and the post-radiotherapy head MRI showed continued growth of the tumor. This was likely the result of her overall impaired systemic immunity and local immunosuppression secondary to the large size of her glioblastoma. Her trilineage dysfunction in hematopoiesis also prevented her from taking temozolomide or bevacizumab during radiation. Therefore, patients with advanced MDS may be better served in hospice at the time of glioblastoma diagnosis.

\section{Acknowledgements}

We thank Elizabeth Kelliher, NP, from the Hematologic Malignancy Service for her care of the patient.

\section{Statement of Ethics}

Written informed consent was obtained from the patient's son after the patient's death for the publication of this case report and the accompanying images.

\section{Conflict of Interest Statement}

The authors have no conflicts of interest to declare.

\section{Funding Sources}

There is no funding source or sponsor to report.

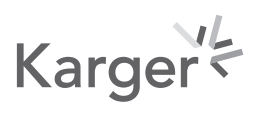




\section{Case Reports in Oncology}

\begin{tabular}{l|l}
\hline Case Rep Oncol 2021;14:424-429 \\
\hline DOI: 10.1159/000513510 & $\begin{array}{l}\text { @ 2021 The Author(s). Published by S. Karger AG, Basel } \\
\text { www.karger.com/cro }\end{array}$ \\
\hline
\end{tabular}

Wong et al.: Myelodysplastic Syndrome Accelerates Glioblastoma Progression

\section{Author Contributions}

E.T.W. and E.J.U. contributed substantially to the conception and design of the case report, as well as to the acquisition, analysis and interpretation of the patient data. E.T.W. drafted the manuscript. E.J.U., J.M., and L.H. critically reviewed and revised the manuscript for important intellectual content and gave their final approval.

\section{References}

1 Stupp R, Taillibert S, Kanner A, Read W, Steinberg DM, Lhermitte B, et al. Effect of tumor-treating fields plus maintenance temozolomide vs. maintenance temozolomide alone on survival in patients with glioblastoma: a randomized clinical trial. JAMA. 2017;318(23):2306-16.

2 Levin VA, Mendelssohn ND, Chan J, Stovall MC, Peak SJ, Yee JL, et al. Impact of bevacizumab administered dose on overall survival of patients with progressive glioblastoma. J Neurooncol. 2015;122(1):145-50.

3 Woroniecka KI, Rhodin KE, Chongsathidkiet P, Keith KA, Fecci PE. T-cell dysfunction in glioblastoma: applying a new framework. Clin Cancer Res. 2018;24(16):3792-802.

4 Ho GK, Uhlmann EN, Wong ET, Uhlmann EJ. Leukopenia is a biomarker for effective temozolomide dosing and predicts overall survival of patients with glioblastoma. Mol Clin Oncol. 2020;13(6):80.

5 Montalban-Bravo G, Garcia-Manero G. Myelodysplastic syndromes: 2018 update on diagnosis, risk-stratification and management. Am J Hematol. 2018;93(1):129-47.

6 Krombach J, Hennel R, Brix N, Orth M, Schoetz U, Ernst A, et al. Priming anti-tumor immunity by radiotherapy: dying tumor cell-derived DAMPs trigger endothelial cell activation and recruitment of myeloid cells. Oncoimmunology. 2019;8(1):e1523097.

7 Deng L, Liang H, Xu M, Yang X, Burnette B, Arina A, et al. STING-dependent cytosolic DNA sensing promotes radiation-induced type I interferon-dependent antitumor immunity in immunogenic tumors. Immunity. 2014;41(5):843-52.

8 Sistigu A, Yamazaki T, Vacchelli E, Chaba K, Enot DP, Adam J, et al. Cancer cell-autonomous contribution of type I interferon signaling to the efficacy of chemotherapy. Nat Med. 2014;20(11):1301-9.

9 Burnette BC, Liang H, Lee Y, Chlewicki L, Khodarev NN, Weichselbaum RR, et al. The efficacy of radiotherapy relies upon induction of type I interferon-dependent innate and adaptive immunity. Cancer Res. 2011;71(7): 2488-96.

10 Stupp R, Hegi ME, Mason WP, van den Bent MJB, Taphoorn MJ, Janzer RC, et al. Effects of radiotherapy with concomitant and adjuvant temozolomide versus radiotherapy alone on survival in glioblastoma in a randomised phase III study: 5-year analysis of the EORTC-NCIC trial. Lancet Oncol. 2009;10(5):459-66.

11 Chongsathidkiet P, Jackson C, Koyama S, Loebel F, Cui X, Farber SH, et al. Sequestration of T cells in bone marrow in the setting of glioblastoma and other intracranial tumors. Nat Med. 2018;24(9):1459-68.

12 Busch MP, Valinsky JE, Paglieroni T, Prince HE, Crutcher GJ, Gjerset GF, et al. Screening of blood donors for idiopathic CD4+ T-lymphocytopenia. Transfusion. 1994;34(3):192-7.

13 Grossman SA, Ye X, Lesser G, Sloan A, Carraway H, Desideri S, et al. Immunosuppression in patients with highgrade gliomas treated with radiation and temozolomide. Clin Cancer Res. 2011;17(16):5473-80.

14 Wong ET, Swanson KD. Dexamethasone - friend or foe for patients with glioblastoma? JAMA Neurol. 2019; 76(3):247-8.

15 Chinnaiyan P, Won M, Wen PY, Rojiani AM, Werner-Wasik M, Shih HA, et al. A randomized phase II study of everolimus in combination with chemoradiation in newly diagnosed glioblastoma: results of NRG Oncology RTOG 0913. Neuro Oncol. 2018;20(5):666-73.

16 Talati C, Sallman D, List A. Lenalidomide: myelodysplastic syndromes with $\operatorname{del}(5 q)$ and beyond. Semin Hematol. 2017;54(3):159-66.

17 Lu L, Payvandi F, Wu L, Zhang LH, Hariri RJ, Man HW, et al. The anti-cancer drug lenalidomide inhibits angiogenesis and metastasis via multiple inhibitory effects on endothelial cell function in normoxic and hypoxic conditions. Microvasc Res. 2009;77(2):78-86. 